\title{
Automated External Defibrillator Issues and Challenges in Saudi Arabia: Knowledge Assessment among Laypersons; Public Safety Perspective
}

Samer A. Al Haliq ( $\square$ saalhaliq@iau.edu.sa )

Imam Abdulrahman Bin Faisal University https://orcid.org/0000-0001-5821-5539

Omar M. Khraisat

Al-Ahliyya Amman University

Mohamed A. Kandil

Imam Abdulrahman Bin Faisal University

Mohammed A. Al Jumaan

Imam Abdulrahman Bin Faisal University College of Medicine

Faris M. Alotaibi

Imam Abdulrahman Bin Faisal University

Fahad S. Alsaqabi

Imam Abdulrahman Bin Faisal University

Hussain M. Alajmi

Imam Abdulrahman Bin Faisal University

Hany A. Ellouly

Imam Abdulrahman Bin Faisal University

Mahmoud A. Al-Haliq

The Hashemite University

Abdullah Alkhawaldeh

Jerash University

Mohammed ALBashtawy

Al al-Bayt University

Sawsan H. Abuhammad

Jordan University of Science and Technology

Research article

Keywords: Automated External Defibrillator, Cardiopulmonary Resuscitation, Layperson, Out of Hospital Cardiac Arrest, Saudi Arabia.

Posted Date: March 30th, 2020 
DOI: https://doi.org/10.21203/rs.2.16101/v2

License: (c) (1) This work is licensed under a Creative Commons Attribution 4.0 International License. Read Full License 
The authors have withdrawn this preprint from Research Square 